The Institute of Space and Astronautical Science

Report SP No.14, December 2000

\title{
The ESA FIRST Cornerstone Mission
}

\author{
By \\ Göran L. PilbratT*
}

(November 1, 2000)

\begin{abstract}
FIRST, the 'Far InfraRed and Submillimetre Telescope', is the fourth cornerstone mission in the European Space Agency (ESA) science programme. It will perform imaging photometry and spectroscopy in the far-infrared and submillimetre part of the spectrum, covering approximately the $60-670 \mu \mathrm{m}$ range.

FIRST will carry a 3.5 metre diameter passively cooled telescope. The science payload complement - two cameras/medium resolution spectrometers (PACS and SPIRE) and a very high resolution heterodyne spectrometer (HIFI) - will be housed in a superfluid helium cryostat. FIRST will be placed in a transfer trajectory towards its operational orbit around the Earth-Sun L2 point by an Ariane 5 (shared with Planck) in early 2007. Once operational FIRST will offer a minimum of 3 years of routine observations; roughly $2 / 3$ of the available observing time is open to the general astronomical community through a standard competitive proposal procedure.
\end{abstract}

\section{INTRODUCTION}

The 'Far InfraRed and Submillimetre Telescope' (FIRST) is a multi-user 'observatory type' mission that targets approximately the $60-670 \mu \mathrm{m}$ wavelength range in the far-infrared and submillimetre part of the electromagnetic spectrum, providing observation opportunities for the entire astronomical community. FIRST is the fourth of the 'cornerstone' missions in the ESA science 'Horizon 2000' plan.

FIRST is the only space facility dedicated to the submillimetre and far-infrared part of the spectrum. Its vantage point in space provides several decisive advantages. The telescope will be passively cooled, which together with a low emissivity and the total absence of (even residual) atmospheric emission offers a very low and stable background that enables very sensitive photometric observations. Furthermore, the absence of atmospheric absorption gives full access to the entire range of this elusive part of the spectrum, which offers the capability to perform completely uninterrupted spectral surveys.

* The author is the FIRST Project Scientist. Address: ESA Astrophysics Division, Space Science Department, ESTEC / SCI-SA, Keplerlaan 1, NL-2201 AZ Noordwijk, The Netherlands; gpilbratt@astro.estec.esa.nl 


\section{SCIENCE OBJECTIVES}

The FIRST science objectives (cf. Rowan-Robinson et al. 1997; Pilbratt 2000) target the 'cold' universe; black-bodies with temperatures between $5 \mathrm{~K}$ and $50 \mathrm{~K}$ peak in the FIRST wavelength range, and gases with temperatures between $10 \mathrm{~K}$ and a few hundred $\mathrm{K}$ emit their brightest molecular and atomic emission lines here. Broadband thermal radiation from small dust grains is the most common continuum emission process in this band. These conditions are widespread everywhere from within our own solar system to the most distant reaches of the Universe!

FIRST - being a unique facility in many ways - has the potential of discovering the earliest epoch proto-galaxies, revealing the cosmologically evolving AGN-starburst symbiosis, and unraveling the mechanisms involved in the formation of stars and planetary system bodies. The key science objectives emphasize specifically the formation of stars and galaxies, and the interrelation between the two. Example observing programmes with FIRST will include:

- Deep extragalactic broadband photometric surveys in the 100-600 $\mu \mathrm{m}$ FIRST 'prime' wavelength band and related research. The main goals will be a detailed investigation of the formation and evolution of galaxy bulges and elliptical galaxies in the first third of the present age of the Universe.

- Follow-up spectroscopy of especially interesting objects discovered in the survey. The far-infrared/submillimetre band contains the brightest cooling lines of interstellar gas, which give very important information on the physical processes and energy production mechanisms (e.g. AGN vs. star formation) in galaxies.

- Detailed studies of the physics and chemistry of the interstellar medium in galaxies, both locally in our own Galaxy as well as in external galaxies, by means of photometric and spectroscopic surveys and detailed observations. This includes implicitly the important question of how stars form out of molecular clouds in various environments.

- Observational astrochemistry (of gas and dust) as a quantitative tool for understanding the stellar/interstellar lifecycle and investigating the physical and chemical processes involved in star formation and early stellar evolution in our own Galaxy. FIRST will provide unique information on most phases of this lifecycle.

- Detailed high resolution spectroscopy of a number of comets and the atmospheres of the cool outer planets and their satellites.

All astronomy missions and observatories - ground, air, and space based - to varying degrees rely on, and complement, each other; in this respect FIRST is not an exception. A major strength of FIRST is its photometric mapping capability for performing unbiased surveys related to galaxy and star formation. Redshifted ultraluminous IRAS galaxies (with SEDs that 'peak' in the 50-100 $\mu \mathrm{m}$ range in their rest frames) as well as class 0 protostars peak in the FIRST 'prime' band; cf. Fig. 1. FIRST is also well equipped to perform spectroscopic follow-up observations to further characterize particularly interesting individual objects.

From past experience, it is also clear that the 'discovery potential' is significant when a new capability is being implemented for the first time. Observations have never been performed in space in the 'prime' band of FIRST. The total absence of (even residual) atmospheric effects - enabling both a much lower background for photometry and full wavelength coverage for 

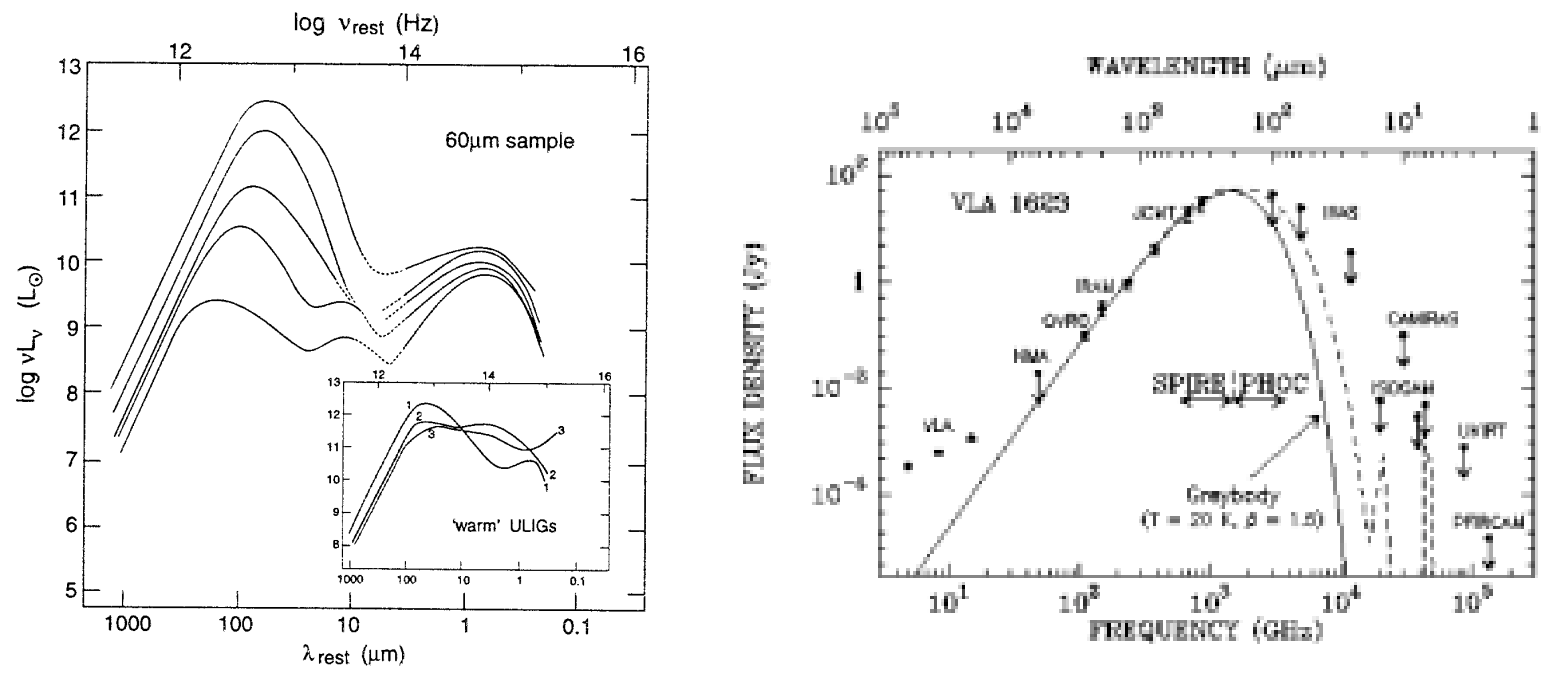

Fig. 1: The FIRST wavelength coverage is ideally suited to for observing redshifted luminous IRAS galaxies (left) and class 0 protostars (right). Observations with PACS and/or SPIRE will enable the bolometric luminosity of such objects to be determined, which is not possible by observing e.g. in the near-infrared or submillimetre only.

spectroscopy - and a cool low emissivity telescope open up a new part of the phase-space of observations. Thus, a space facility is essential in this wavelength range and FIRST will be breaking new ground!

\section{TELESCOPE AND SCIENCE PAYLOAD}

In order to fully exploit the favourable conditions offered by being in space FIRST will need a precise, stable, very low background telescope, and a complement of very sensitive scientific instruments. The FIRST telescope will be passively cooled - to maximize size - while the instruments will be housed inside a superfluid helium cryostat.

\subsection{Telescope Development}

The FIRST telescope must have a total wavefront error (WFE) of less than $10 \mu \mathrm{m}$ (with a goal of $6 \mu \mathrm{m}$ - corresponding to 'diffraction-limited' operation at $150 \mu \mathrm{m}$ (goal $90 \mu \mathrm{m})$ - in orbit, and a very low emissivity. Being protected by a fixed sunshade, it will radiatively cool to an operational temperature of around $80 \mathrm{~K}$ in orbit. The present planning assumes that this telescope will be provided by NASA.

The baseline is a Ritchey-Chrétien design with a $3.5 \mathrm{~m}$ diameter primary and an 'undersized' secondary. The telescope has a segmented primary mirror made of carbon fibre reinforced plastic (CFRP) structure, with a zerodur secondary precisely machined to correct for low spatial frequency imperfections in the primary. An aggressive development programme is underway (Connell et al. 2000) to optimize the design - including optical, mechanical, thermal, and straylight properties - perfect manufacturing and testing procedures, and control potential detrimental environmental impacts. 


\subsection{Scientific Instruments}

The FIRST science payload has been conceived and optimized with the prime science goals in mind, but in addition it offers a wide range of capabilities for the 'general' observer. It was selected on the basis of the response to an Announcement of Opportunity (AO) issued in October 1997. The Principal Investigators (PIs) and the instruments selected were:

- The Photoconductor Array Camera and Spectrometer (PACS) instrument will be built by a consortium led by A. Poglitsch, MPE, Garching, Germany.

- The Spectral and Photometric Imaging REceiver (SPIRE) instrument will be built by a consortium led by M. Griffin, QMW, London, UK.

- The Heterodyne Instrument for FIRST (HIFI) instrument will be built by a consortium led by Th. de Graauw, SRON, Groningen, The Netherlands.

The PI consortia provide the instruments to ESA under their own funding, in return for guaranteed observing time. The scientific payload complement was selected by the ESA Science Programme Committee in May 1998 and approved in February 1999.

PACS (cf. Poglitsch et al. 2000) is an imaging photometer and integral field line spectrometer for wavelengths up to $\sim 210 \mu \mathrm{m}$. It has recently been redesigned to employ in total four detector arrays, two bolometer arrays for photometry, and two Ge:Ga detector arrays for spectroscopy (cf. Fig. 2).
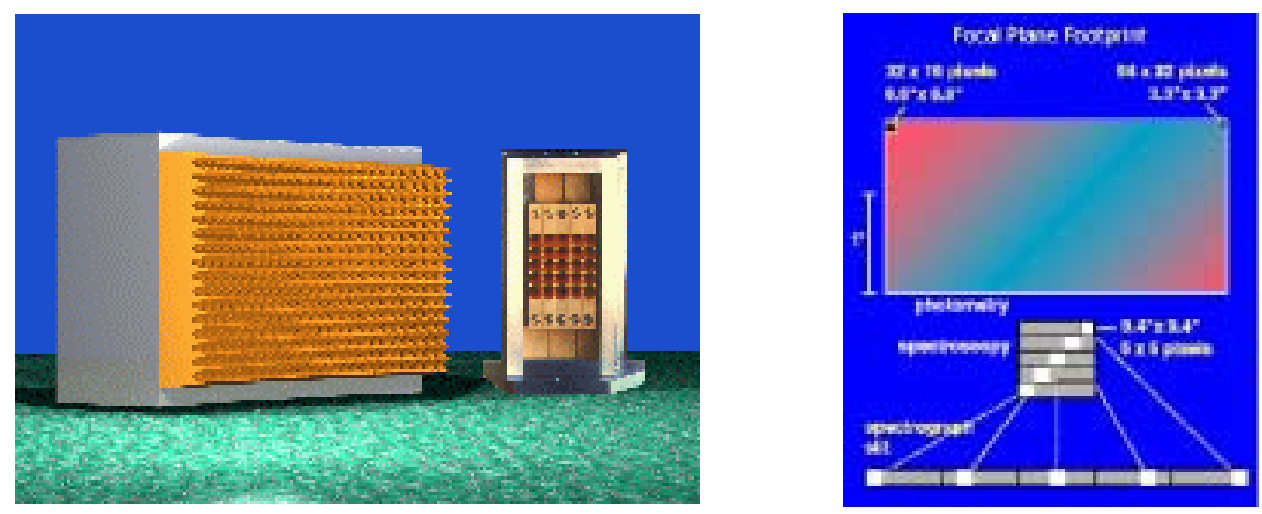

Fig. 2: Left: A computer rendering of a PACS $25 \times 16$ Ge:Ga array compared with the $5 \times 5$ Kuiper FIFI array. Right: The PACS focal plane footprint.

PACS has three photometric bands with $R \sim 2$. The short wavelength 'blue' array covers the $60-90$ and $90-130 \mu \mathrm{m}$ bands, while the 'red' array covers the $130-210 \mu \mathrm{m}$ band. In photometric mode one of the 'blue' bands and the 'red' band are observed simultaneously. The bolometer arrays fully sample a field of view of $1 ! 75 \times 3.5$, and provide a point source detection limit of $\sim 3 \mathrm{mJy}\left(5 \sigma, 1\right.$ hour). An internal ${ }^{3} \mathrm{He}$ sorption cooler will provide the $300 \mathrm{mK}$ environment needed by the bolometers.

For spectroscopy PACS covers $57-210 \mu \mathrm{m}$ in three contiguous bands, providing a velocity resolution in the range $150-200 \mathrm{~km} \mathrm{~s}^{-1}$ and an instantaneous coverage of $\sim 1500 \mathrm{~km} \mathrm{~s}^{-1}$. The 
two Ge:Ga arrays are appropriately stressed and operated at slightly different temperatures - cooled by being 'strapped' to the liquid helium - in order to optimize sensitivity for their respective wavelength coverage. The point source detection limit is $\sim 3 \times 10^{-18} \mathrm{~W} \mathrm{~m}^{-2}$.

SPIRE (cf. Griffin et al. 2000) comprises an imaging photometer and a symmetrical MachZender imaging spectrometer. SPIRE has five bolometer detector arrays (cf. Fig. 3). They employ spider-web bolometers with NTD Ge temperature sensors, with each pixel being fed by a single-mode $2 \mathrm{~F} \lambda$ feedhorn, and JFET readout electronics. The bolometers are cooled to $300 \mathrm{mK}$ by an internal ${ }^{3} \mathrm{He}$ sorption cooler.
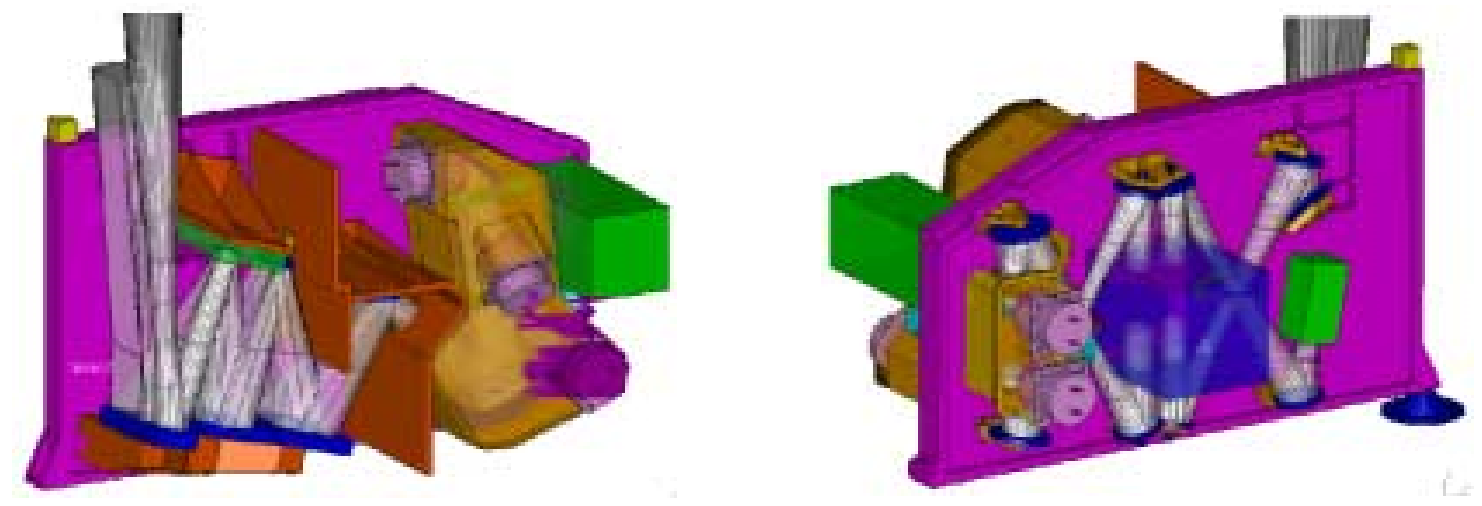

Fig. 3: Left: The SPIRE photometer, its three bolometer arrays are towards the right. Right: The spectrometer, in the middle the mirror mechanism box, and on the left its two bolometer arrays. All five detector arrays are situated close to the internal ${ }^{3} \mathrm{He}$ sorption cooler which provides the $300 \mathrm{mK}$ operating temperature.

SPIRE has been designed to maximize mapping speed. In its broadband $(R \sim 3)$ photometry mode it simultaneously images a $4^{\prime} \times 8^{\prime}$ field on the sky in three colours centred on 250, 350, and $500 \mu \mathrm{m}$. Since the telescope beam is not instantaneously fully sampled, it will be required either to scan along a preferred angle, or to 'fill in' by 'jiggling' with the internal beam steering mirror.

The SPIRE spectrometer is based on a Mach-Zender configuration with novel broad-band beam dividers. Both input ports are used at all times, the signal port accepts the beam from the telescope while the second port accepts a signal from a calibration source, the level of which is chosen to balance the power from the telescope in the signal beam. The two output ports have detector arrays dedicated for 200-300 and 300-600 $\mu \mathrm{m}$ respectively. The maximum resolution will be in the range 100-1000 at a wavelength of $250 \mu \mathrm{m}$, and the field of view $\sim 2.6^{\prime}$.

HIFI (cf. Whyborn 1997) is a very high resolution heterodyne spectrometer. It offers velocity resolution in the range $0.3-300 \mathrm{~km} \mathrm{~s}^{-1}$, combined with low noise detection using superconductor-insulator-superconductor (SIS) and hot electron bolometer (HEB) mixers. HIFI is not an imaging instrument, it provides a single pixel on the sky.

The focal plane unit (FPU, cf. Fig. 4), houses seven mixer assemblies, each one equipped with two orthogonally polarized mixers. Bands $1-5$ utilize SIS mixers that together cover approximately $500-1250 \mathrm{GHz}$ without any gaps in the frequency coverage. Bands 6 and 7 
utilize HEB mixers, and target the 1410-1910 GHz band. The FPU also houses the optics that feeds the mixers the signal from the telescope and combines it with the appropriate local oscillator (LO) signal, as well as provides a chopper and the capability to view internal calibration loads.

The LO signal is generated by a source unit located in the spacecraft service module (SVM, cf. Section 4). By means of waveguides it is fed to the LO unit, located on the outside of the cryostat vessel, where it is amplified, multiplied and subsequently quasioptically fed to the FPU. The SVM also houses the spectrometers.
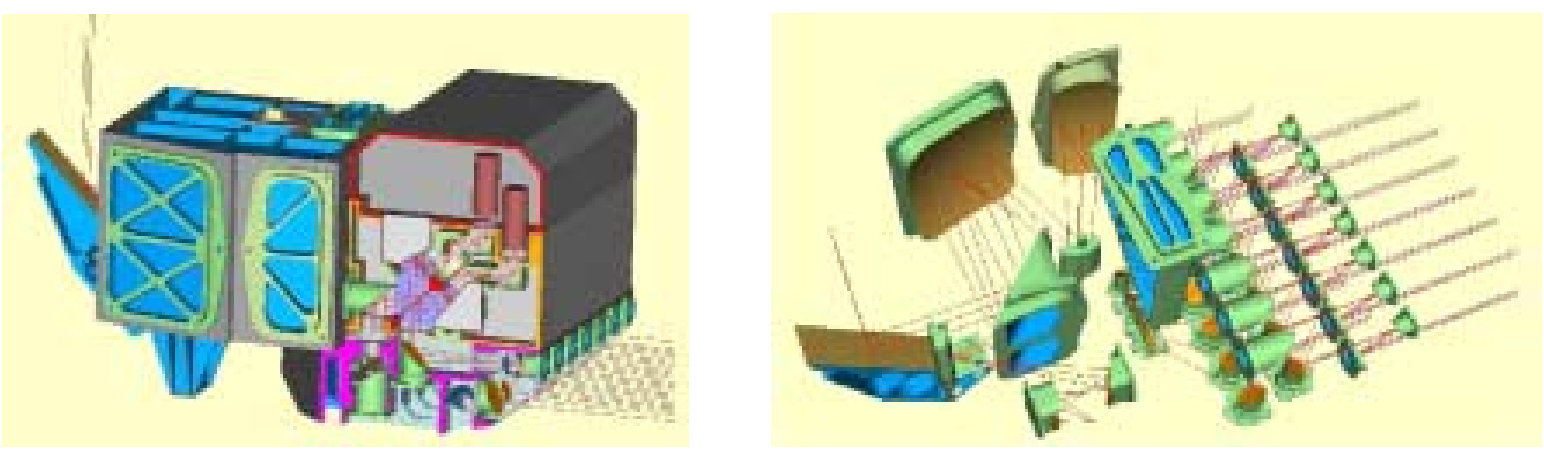

Fig. 4: The HIFI focal plane unit. Left: The M3 mirror picks up the signal from the telescope and feeds it into the common optics unit, then into the mixer unit where it is combined with the local oscillator signal and fed to the mixers. Right: An exploded view of the optics.

\section{SPACECRAFT AND ORBIT}

The FIRST configuration shown in Fig. 5 (cf. Passvogel \& Felici 2000) envisages a payload module based on the now well proven ISO cryostat technology. This configuration has been used to establish payload interfaces and study mission design. It is modular, consisting of a payload module (PLM, cf. Collaudin et al. 2000), comprising the superfluid helium cryostat - housing the optical bench with the instrument FPUs - which supports the telescope, star trackers, and some payload associated equipment; and the service module (SVM), which provides the 'infrastructure' and houses the 'warm' payload electronics.

This FIRST concept measures approximately $9 \mathrm{~m}$ in height, $4.5 \mathrm{~m}$ in width, and has an approximate launch mass of $3200 \mathrm{~kg}$. The $3.5 \mathrm{~m}$ diameter FIRST telescope is protected by the sunshade, and will cool passively to around $80 \mathrm{~K}$. The FIRST science payload focal plane units are housed inside the cryostat, which contains superfluid helium at $1.65 \mathrm{~K}$. Fixed solar panels on the sunshade deliver $1 \mathrm{~kW}$ power. Three star-trackers in a skewed configuration and the local oscillator unit for the heterodyne instrument are visible on the outside of the cryostat vacuum vessel. The mating adaptor remains attached to FIRST after separation.

An Ariane 5 launcher shared by the ESA CMB mapping mission Planck and FIRST will inject both satellites into a transfer trajectory towards the second Lagrangian point (L2) in the Sun-Earth system. They will then separate from the launcher, and subsequently operate independently from orbits of different amplitude around L2.

The L2 point is situated 1.5 million $\mathrm{km}$ away from the Earth in the anti-sunward direction. 

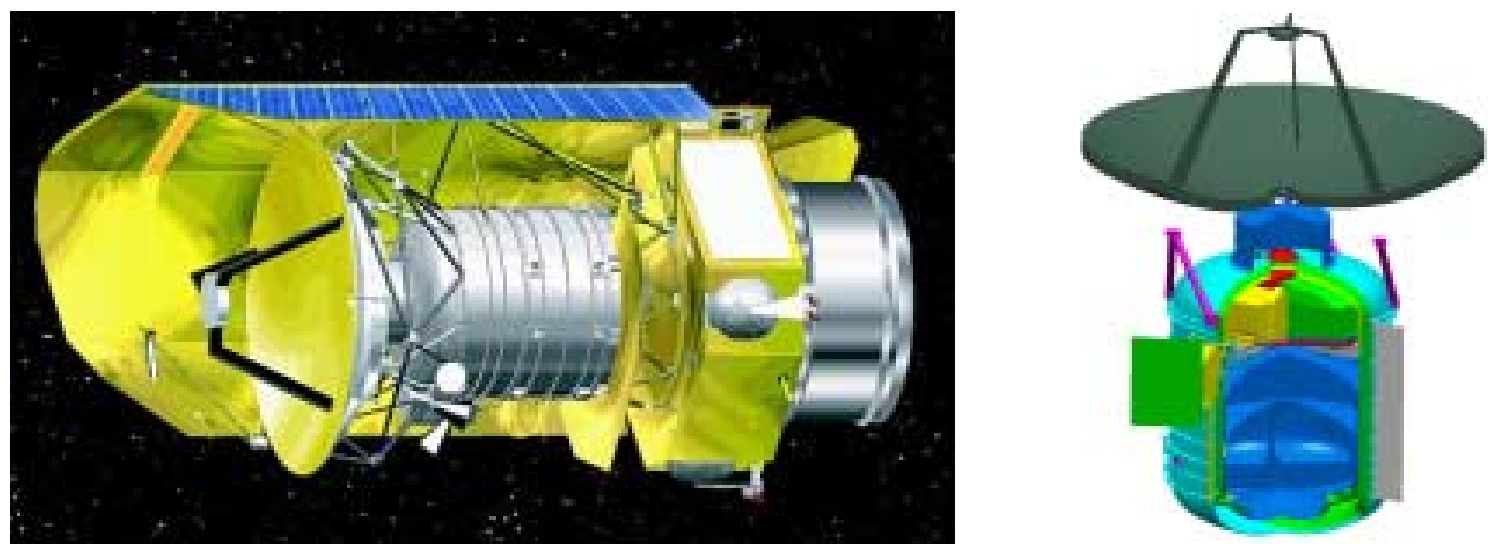

Fig. 5: Left: Artist's view of the FIRST satellite in orbit, see text. Right: Exploded view of the FIRST PLM showing the three instrument focal plane units on the optical bench on top of the superfluid helium tank inside the cryostat.

It offers a stable thermal environment with good sky visibility. Since FIRST will be in a large orbit around L2, which has the advantage of not costing any 'orbit injection' $\Delta v$, its distance to the Earth will vary between 1.2 and 1.8 million $\mathrm{km}$. The transfer to the operational orbit will last approximately 4 months. After cooldown and outgassing have taken place, it is planned to use this time for commissioning and performance verifications. Once these crucial mission phases have been successfully accomplished, FIRST will go into the routine science operations phase for a minimum duration of 3 years.

\section{SCIENCE OPERATIONS}

FIRST will be a multi-user observatory open to the general astronomical community. The observation time will be shared between guaranteed and open time. The guaranteed time (approximately one third of the total time) is 'owned' by contributors to the FIRST mission (mainly by the PI instrument consortia), whereas the open time will be allocated to the general community (including the guaranteed time holders) on the basis of calls for observing time. A small amount of the open time (discretionary time) will be reserved for targets that could not have been foreseen at the time of a proposal deadline.

The scientific operations of FIRST will be conducted in a novel 'decentralised' manner. The proposed ground segment concept (cf. also Bauer et al. 1998) comprises five elements:

- a FIRST Science Centre (FSC), provided by ESA,

- three dedicated Instrument Control Centres (ICCs), one for each instrument, provided by their PIs,

- a Mission Operations Centre (MOC), provided by ESA.

In addition it is foreseen that the NASA FIRST Science Center at the Infrared Processing and Analysis Center (IPAC) will become the sixth element.

The FSC acts as the single-point interface to the science community and outside world in general. The FSC provides information and user support related to the entire life-cycle of an 
observation, from calls for observing time, the proposing procedure, proposal tracking, data access and data processing, as well as general and specific information about 'using' FIRST and its instruments.

All scientific data will be archived and made available to the data owners. After the proprietary time has expired for a given data set, these data will be available to the entire community in the same manner they were previously available only to the original owner.

\section{STATUS AND SCHEDULE}

FIRST is presently in a pre-phase B development phase. Industrial studies have been carried out to define payload and telescope interfaces, and to refine the cryostat design. The instrument consortia are in the process of finalizing the instrument designs in order to start building the first test models. The first formal review cycle, the instrument science verification review (ISVR), has been successfully conducted.

The Invitation to Tender (ITT) to industry for phases B/C/D/E will be issued on 1 September 2000, and phase B - the detailed design phase - will start in June 2001, after a prime contractor has been selected. The current planning (cf. Passvogel \& Felici 2000) envisages a series of milestones, including instrument and telescope flight model deliveries in 2004, leading to a launch early in the year 2007.

Additional information - including online versions of some of the references listed below can be found on the ESA Astrophysics FIRST World Wide Web site at the following URL: http://astro.estec.esa.nl/FIRST/.

\section{ACKNOWLEDGMENTS}

This paper has been written on behalf of the large number of people who either currently are working on one or more of the many aspects of the FIRST mission - in ESA, the scientific community, and industry - or who have been doing so in the past.

\section{REFERENCES}

Bauer, O. H. et al. 1998, SPIE Proc., 3349, 312

Collaudin, B. et al. 2000, SPIE Proc., 4013, 161

Connell, S. J. et al. 2000, SPIE Proc., 4013, 173

Griffin, M., Swinyard, B. M., \& Vigroux, L. G. 2000, SPIE Proc., 4013, 184

Passvogel, Th. \& Felici, F. 2000, SPIE Proc., 4013, 152

Pilbratt, G.L. 2000, SPIE Proc., 4013, 142

Poglitsch, A., Geis, N., \& Waelkens, C. 2000, SPIE Proc., 4013, 221

Rowan-Robinson, M., Pilbratt, G., \& Wilson, A. (ed.) 1997, ESA SP-401, The Far InfraRed and Submillimetre Universe (Noordwijk: ESA)

Whyborn, N. D. 1997, in: ESA SP-401, The Far InfraRed and Submillimetre Universe, ed. M. RowanRobinson, G. Pilbratt, \& A. Wilson (Noordwijk: ESA), 19 